\title{
Is temporal order encoded automatically?
}

\author{
ROSE T. ZACKS \\ Michigan State University, East Lansing, Michigan \\ LYNN HASHER \\ Temple University, Philadelphia, Pennsylvania \\ JOSEPH W. ALBA \\ University of Florida, Gainesville, Florida \\ HENRIANNE SANFT \\ Carnegie-Mellon University, Pittsburgh, Pennsylvania \\ and \\ KAREN CANIGLIA ROSE \\ Temple University, Philadelphia, Pennsylvania
}

\begin{abstract}
The reported experiment tested the suggestion that encoding of temporal order is automatic. Specifically, two of Hasher and Zacks's (1979) automaticity criteria were examined: (1) that the amount and appropriateness of practice received would not affect acquisition of temporal information, and (2) that reliable individual differences would not be found on a test of memory for temporal order. Contrary to expectations, neither of these criteria was confirmed: Retention of temporal order increased with practice at three (or four) successive lists. And, reliable individual differences were indicated by the findings that subjects' relative performance levels remained stable across lists, and that groups with higher average academic ability outperformed those with lower ability. Similar results were obtained for a free-recall task (in which case they were expected). Problems of assessing degrees of nonautomaticity are discussed. Our data are seen to be in general conformity with Tzeng's (e.g., Tzeng \& Cotton, 1980) "study-phase" retrieval theory of temporal coding.
\end{abstract}

It has been argued that temporal order information plays a central role in normal memory functioning (e.g., Hirst, 1982; Tulving \& Madigan, 1970; Tzeng, Lee, \& Wetzel, 1979). For example, temporal codes are presumed to limit interlist interference and to allow subjects to recall a specified list of items when multiple lists have been presented (Tulving \& Madigan, 1970). One way to summarize such suggestions is through the concept of "list differentiation" (Underwood, 1949). A related process, that of "updating," has recently been investigated by Bellezza (1982). His research suggests that when distinctive temporal cues are available, subjects are able to use these cues to limit the search set and thereby to minimize proactive interference. These findings and those of others (e.g., Winograd, 1968) point to a heavy reliance on temporal information.

We assume that processes such as list differentiation and updating depend upon temporal order information that is directly represented in memory-for example, by time tags or contextual associations (cf. Tulving \&

This research was supported by National Institute of Mental Health Grant MH 33140. The authors are grateful for the able assistance of Clarla Clos. Requests for reprints should be sent to Rose Zacks, Department of Psychology, Michigan State University, East Lansing, MI 48824.
Madigan, 1970; Tzeng \& Cotton, 1980). The major alternative to this view proposes that subjects, not having stored temporal information in memory, make temporal judgments on the basis of the relative "strengths" of memory traces (e.g., Hinrichs, 1970). This view receives little empirical support. For example, it cannot account for the fact that the "strong" primacy items of a list are not misjudged as being more recent than the "weaker" middle items or for Tzeng and Cotton's (1980) finding that within-category judgments of temporal order are more accurate than between-category judgments. Thus, it seems legitimate to speak of temporal codes being stored.

This paper addresses a specific question in regard to the processing of temporal information: Are temporal codes stored automatically? A number of different investigators have hypothesized that they are (e.g., Hasher \& Zacks, 1979; McCormack, 1981; Toglia \& Kimble, 1976; Tulving \& Madigan, 1970; Zimmerman \& Underwood, 1968), but it must be admitted that the support for the hypothesis of automaticity is limited. In fact, it is not a simple matter to provide support for an automaticity hypothesis (e.g., Fisk, Derrick, \& Schneider, 1981; Navon \& Gopher, 1979). In the present research, we adopted an approach that has proved useful in the study of encoding of frequency-of-occurrence 
information (Hasher \& Zacks, 1979, in press; Zacks, Hasher, \& Sanft, 1982). The approach employs multiple criteria of automaticity derived from a general framework for studying memory encoding processes.

Our framework assumes that encoding processes differ in the mental capacity they require and that individuals vary in systematic ways in the capacity they have available (Hasher \& Zacks, 1979, in press). The automatic processes are those that require the minimum amount of capacity; these function continuously at optimum levels, irrespective of the intentions or strategies of the individual. These ideas support six empirical criteria of automaticity, all of which must be confirmed to conclusively establish that a process is automatic. Thus, to show that one type of information is encoded automatically requires the following criteria: (1) the information is encoded without intention; (2) the addition of intention does not improve the efficacy of the encoding process; (3) training has no effect; (4) there are minimal individual differences in the encoding of the information; (5) similarly, there are minimal age differences; and, finally, (6) the encoding of the information is not disrupted by factors that reduce cognitive capacity (e.g., illness, depression) or by competing cognitive demands.

In the case of frequency-of-occurrence information, there is confirmatory evidence with regard to each of these criteria (see Hasher \& Zacks, in press), and thus there is a firm empirical base for arguing that frequency of occurrence is encoded automatically. In the case of temporal order information, the available data are more limited, but some earlier findings are supportive. For one thing, temporal information is known to be stored under incidental learning conditions (Criterion 1: e.g., Hintzman \& Block, 1971, 1973; Proctor \& Ambler, 1975). The addition of an intentional instruction appears not to improve upon the temporal knowledge that is acquired incidentally (Criterion 2: Toglia \& Kimble, 1976; Zimmerman \& Underwood, 1968). In addition, temporal information is processed without cost to other ongoing activity; for example, preparation for a temporal task does not disrupt preparation for a recall test (Criterion 6: Zimmerman \& Underwood, 1968). Finally, the ability to encode temporal information is known to be present for very young children (Criterion 5: e.g., Brackbill \& Fitzgerald, 1972), and it may not improve during childhood (Brown, 1973) or decline during old age (McCormack, 1981; Perlmutter, Metzger, Nezworski, \& Miller, 1981; but see McCormack, 1982).

The experiment reported here considered two of the automaticity criteria: the effects of practice (Criterion 3 ) and individual differences (Criterion 4). Under the automaticity hypothesis, the encoding of temporal order information should not be affected by the amount or appropriateness of practice, or by individual differences. As a counterpoint to the null effects predicted for the temporal task, another task was also used in the study. The latter, free recall, was expected to show significant practice effects and reliable individual differences (see also Zacks et al., 1982).

Generally, it is presumed that practice benefits memory processing through mechanisms such as the acquisition of effective mnemonic strategies or the reduction of the amount of capacity required (e.g., Bransford, 1979). Since automatic processes require minimal capacity and since they are unaffected by the use of strategies (Hasher \& Zacks, 1979, in press), neither the degree nor the appropriateness of practice should influence them. As a study of practice effects in the encoding of temporal information, this experiment required subjects to make temporal order judgments after each of three successive lists. On a fourth list, the temporal judgment performance of these trained subjects was compared with that of subjects with three trials of free-recall training. Findings of no change in performance over three lists on the temporal task and no effect of test-appropriate versus test-inappropriate training on the final list would indicate automaticity of encoding of temporal information. These same variables (practice on successive lists and transfer from the same vs. a different memory task) were also studied for single-trial free recall. On this task, performance depends heavily on the use of capacitydemanding, mnemonic strategies (including organization and rehearsal). The efficiency of such strategies (Hasher \& Zacks, 1979, called them "effortful processes") increases with practice. Therefore, recall was expected to increase over lists, and performance on List 4 was expected to show the benefit of test-appropriate training.

A similar argument can be made with respect to individual differences. Automatic encoding operations require very little capacity, and therefore individual variations in the availability or deployment of mental capacity (as affected by, for example, motivation, stress, or experience) should have little influence. And the insensitivity of automatic processing to strategies means that variables correlated with the use of mnemonic strategies (e.g., education, intellectual ability) should also have no effect. By contrast, effortful cognitive operations such as those used for free-recall tasks are known to be influenced by training, experience, and motivation. Stable and sizable individual differences were thus expected on the free-recall task but not on the temporal judgment task.

This experiment allowed us to examine the presence of individual differences in two ways: (1) by looking for stable versus unstable rankings of subjects' performance across the three or four successive tests of either recall or temporal judgment; and (2) by comparing the performance on the two tasks of groups of students who were likely to differ in academic ability. This latter comparison was made possible by testing students at two universities, called here University $X$ and University $Y$; the median verbal and quantitative SAT scores were higher for the students at University $X$ than for the students at University $Y$ by 20 and 80 points, respec- 
tively. ${ }^{1}$ Since a number of performance variables are known to be correlated with SAT scores, these score differentials led to the prediction of superior performance by students at University $\mathrm{X}$ on tasks requiring effortful processing.

It is important to note that the design of the current experiment paralleled that of Experiment 1 in Zacks et al. (1982). The latter experiment compared the encoding of frequency information (an automatic process) to preparing for free recall. Both practice effects and reliable group and individual differences were obtained for free recall; neither type of effect was found for frequency information. This pattern of results is especially important in relation to the experiment reported here because the University $\mathrm{Y}$ subjects of the current study came from the same population as the University B subjects of the Zacks et al. (1982) frequency study. Thus, the results of the earlier study support the validity of the current design for testing the practice and individual difference criteria of automaticity.

\section{METHOD}

\section{Design}

Four groups of subjects from each of the two universities were given a single presentation and a single test trial on each of four lists of words. For any subject, the first three lists always involved the same memory test. Half the subjects had three trials on a temporal order judgment task (T), and the other half had three trials on a free-recall task (R). For List 4, half of the subjects who had been tested with each memory test continued with the same memory test (called groups T-T and R-R), whereas the other half were switched to the alternative test (called groups $T-R$ and $R-T$, where the first letter denotes the test given on the first three trials and the second letter the test on the fourth trial). On all four lists, the subjects were fully informed, prior to list presentation, about the type of memory test they would receive. The experiment thus had eight groups conforming to a 2 (university) $\times 2$ (task on first three lists) $\times 2$ (task on fourth list) design.

\section{Materials}

The experimental materials consisted of four 34-word lists, each from a different category: things associated with eating, things associated with clothing, places or locations, and small things. The items were culled from the Battig and Montague (1969) norms. For each list, 4 of the 34 words were selected at random to serve as never-presented distractors on the temporal task; two presentation orders of the remaining 30 words were generated. Four sequences of the four different categories were used such that each category occurred one-fourth of the time at each position in the practice sequence. With four different sequences of the categories and two different presentation orders for each, there were eight different unique presentation combinations. Each was used with an equal number of subjects in each group. Two unique orders of all 34 words were generated for the temporal judgment test.

\section{Procedure}

The subjects were run in small groups. Although all subjects in a group studied the same sequence of lists, the experimental condition assigned to each subject varied. The subjects were given booklets that contained study and test instructions for each list as well as pages for writing answers on the memory tests. The instructions that the subjects read prior to the presentation of List 1 fully informed them about the memory test that they would receive. The written instructions also reminded the subjects about the type of memory test prior to the presentation of each of the subsequent lists, and, in cases of a change of test on List 4 , the prelist instructions described the new memory test. For a recall test, the instructions stated that the subjects would be asked to recall, in any order, as many words as they could. For a temporal order memory test, the instructions stated that the subject would be asked to write down, next to each word, its serial position.

On free-recall tests, the subjects wrote the recalled material on answer sheets on which appeared 30 blank lines. On the temporal task, the 34 words for the appropriate category were listed down the left-hand side of the page, with a space for a response next to each. The numbers 1 to 30 were used to indicate the temporal order of the words, with the number 1 referring to the first word presented and the number 30 to the last. Words not recognized were to be given 0 s. On each test, the subjects were required to use each number between 1 and 30 once and to use four 0 s. The numbers 1 to 30 were listed on the response page, and the subjects were told to cross out each number as it was used to facilitate complying with the instructions.

Prior to the presentation of each list, its category was announced and then the list was shown at a 5 -sec rate by means of a Kodak Carousel slide projector with an internal timer. Five minutes were allowed for each memory task. After the final list, the subjects in Groups T-T and T-R were asked about any strategies employed on the temporal task.

\section{Subjects}

There were 24 subjects in each of the four University X groups and 16 in each of the four University $Y$ groups. The subjects participated for course credit.

\section{RESULTS}

In order that position effects could be studied, the presentation orders were divided into sixths. An alpha level of .05 is used throughout.

\section{Free Recall}

Lists 1-3. The main effects of university, list number, and sixth of list were all significant. As can be seen in Table 1, University $X$ students recalled more words per list than did University $Y$ students $[F(1,76)=11.86$, $\mathrm{MSe}=6.87$, and performance increased from List 1 to List 2 and then remained stable $[F(2,152)=6.34$, MSe $=1.25]$. Typical free-recall serial position curves were obtained $[\mathrm{F}(5,380)=37.75, \mathrm{MSe}=1.36]$ (see the top half of Figure 1). The significant list $\mathrm{x}$ serial position interaction $[\mathrm{F}(10,760)=1.95, \mathrm{MSe}=1.28]$ reflected the usual (e.g., Hasher, 1973), although here slight, decrease in primacy and increase in recency with succes-

Table 1

Mean Number Recalled (Maximum $=30$ )

\begin{tabular}{lccccc} 
& & & & \multicolumn{2}{c}{ List 4 } \\
\cline { 5 - 6 } & List 1 & List 2 & List 3 & R-R & T-R \\
\hline University X & 18.5 & 20.3 & 20.8 & 21.4 & 16.6 \\
University Y & 16.5 & 17.6 & 16.8 & 17.4 & 15.4 \\
\hline
\end{tabular}

Note-For Lists 1-3, means are based on ns of 48 and 32 for Universities $X$ and $Y$, respectively; for List 4, the corresponding ns are 24 and 16. 


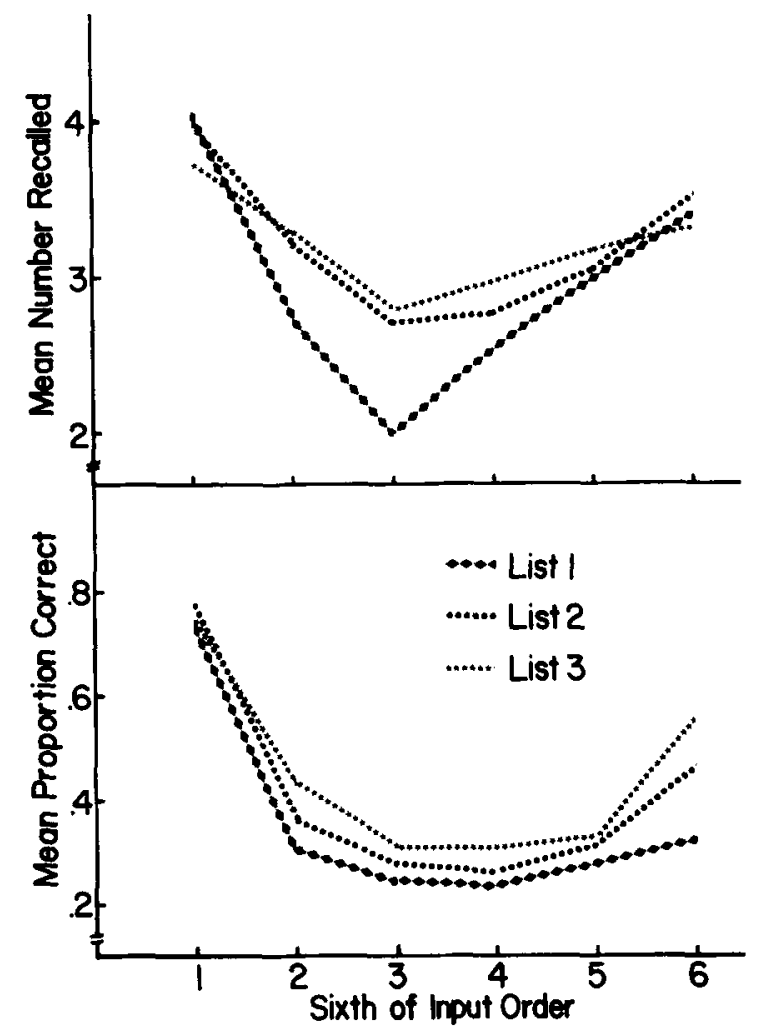

Figure 1. Serial position curves for each of Lists 1-3. Top half displays results for the recall task; bottom half displays results for the correct judgment measure of the temporal task.

sive lists. A second interaction between university and sixth of list resulted from greater bowing of the serial position curve for University $\mathrm{Y}$ than for University $X$ students $[F(5,380)=2.87, \mathrm{MSe}=1.36]$.

List 4. The List 4 performance of Groups R-R and $T-R$ is also shown in Table 1 . Experience at recalling (Group R-R) gave subjects a substantial advantage on List $4[\mathrm{~F}(1,76)=9.87, \mathrm{MSe}=3.62]$. Other significant effects paralleled those of Lists 1-3: University X's advantage was maintained $[F(1,76)=5.94, \mathrm{MSe}=3.62]$, and items in primacy and recency positions were recalled better than others $[F(5,380)=10.64$, MSe $=1.41]$.

These results are all congruent with the assumption that effortful processing is required for good free-recall performance (see also Zacks et al., 1982): (1) Subjects improved with practice; (2) appropriate practice benefited performance; (3) able learners were better than less able learners.

\section{Temporal Judgments}

A judgment of 0 for a given item on the temporal judgment task indicated that the subject believed that item to be new. Any other judgment indicated that the subject believed the item to have been on the presentation list. Because there was very little variability in hits or false alarms, these data are not reported. All analyses of memory for temporal order were performed only on those items correctly recognized as being old.
Some controversy exists about the appropriate measures for estimating memory for temporal order from a temporal judgment task (e.g., Toglia \& Kimble, 1976). We used three measures that jointly span most of the literature on this task: (1) correct judgments, instances in which a subject's judgment was within one item of the actual input position (cf. Underwood \& Malmi, 1978); (2) the (Pearson product-moment) correlation, calculated for each subject, between the judged order and the true order (cf. Tzeng et al., 1979); and (3) mean position judgment for items in each sixth of the list (cf. Hintzman \& Block, 1971). ${ }^{2}$

Correct judgments. Table 2 shows the mean proportions of items assigned to correct input positions. On Lists $1-3$, University $X$ subjects outperformed University $Y$ subjects $[F(1,76)=20.63$, MSe $=.16]$, and performance increased over lists $[F(2,152)=12.42$, MSe $=$ $.08]$. The significant list $\mathrm{x}$ university interaction $[F(2,152)=3.23, \mathrm{MSe}=.08]$ reflects the fact that University $\mathrm{Y}$ subjects showed no improvement until List 3, whereas University $\mathrm{X}$ subjects improved from Lists 1 to 2 as well. Analysis of serial position effects (see the bottom half of Figure 1) indicated reliable primacy and recency effects $[F(5,380)=157.75, \mathrm{MSe}=$ .05] and an inequality across list segments in the amount of improvement over lists $[F(10,760)=3.84$, $\mathrm{MSe}=.04-$ the first sixth of the list showed the least improvement, and the recency effect increased over lists].

On List 4, University $X$ subjects again gave more correct judgments than University $Y$ subjects $[F(1,76)=$ $7.86, \mathrm{MSe}=.21]$, and performance varied over input position $[F(5,380)=49.38, \mathrm{MSe}=.05]$. In addition, the two transfer conditions ( $T-T$ and $R-T$ ) had somewhat different serial position curves $[\mathrm{F}(5,380)=2.45$, $\mathrm{MSe}=$ $.05]$, with Condition T-T showing a larger recency effect than Condition R-T. However, overall performance did not differ as a function of appropriate versus inappropriate practice $(F<1)$.

Correlations between judged and true position. As can be seen from the data in Table 3, the mean correlations were sizable, and the majority of the individual correlations were reliable; again, there is evidence that subjects are sensitive to temporal order information. Contrary to our expectations, however, the correlations (across Lists 1-3) showed a significant increase with lists $[\mathrm{F}(2,152)=5.04$, MSe $=.04]$. The correlations

Table 2

Mean Proportion Correct Temporal Judgments

\begin{tabular}{lccccc} 
& & & & \multicolumn{2}{c}{ List 4 } \\
\cline { 5 - 6 } \cline { 5 - 6 } & List 1 & List 2 & List 3 & T-T & R-T \\
\hline University X & .370 & .463 & .498 & .505 & .473 \\
University Y & .324 & .329 & .380 & .381 & .357 \\
\hline
\end{tabular}

Note-For Lists 1-3, the means are based on ns of 48 and 32 for Universities $X$ and $Y$, respectively; for List 4 , the corresponding ns are 24 and 16. 
Table 3

Mean Pearson Product-Moment Correlations Betweem Judged Order and

True Order and Numbers of Significant Individual Correlations

\begin{tabular}{|c|c|c|c|c|c|c|c|c|c|c|}
\hline & \multirow{2}{*}{\multicolumn{2}{|c|}{ List 1}} & \multirow{2}{*}{\multicolumn{2}{|c|}{ List 2}} & \multirow{2}{*}{\multicolumn{2}{|c|}{ List 3}} & \multicolumn{4}{|c|}{ List 4} \\
\hline & & & & & & & \multicolumn{2}{|c|}{$\mathrm{T}-\mathrm{T}$} & \multicolumn{2}{|r|}{$\mathrm{R}-\mathrm{T}$} \\
\hline & Mean & No. Signif. & Mean & No. Signif. & Mean & No. Signif. & Mean & No. Signif. & Mean & No. Signif. \\
\hline University $X$ & .564 & $43 / 48$ & .676 & $46 / 48$ & .673 & $43 / 48$ & .691 & $22 / 24$ & .634 & $21 / 24$ \\
\hline University $\mathrm{Y}$ & .475 & $26 / 32$ & .450 & $24 / 32$ & .560 & $29 / 32$ & .511 & $13 / 16$ & .469 & $13 / 16$ \\
\hline
\end{tabular}

were also reliably higher for students from University $\mathrm{X}$ than for those from University $Y[F(1,76)=20.60$, $\mathrm{MSe}=.06]$. Furthermore, University $\mathrm{X}$ students benefited more from practice than did University $Y$ students $[\mathrm{F}(2,152)=3.93, \mathrm{MSe}=.04]$. The only significant effect on List 4 was the difference between the two universities $[\mathrm{F}(1,76)=11.24, \mathrm{MSe}=.05]$; appropriateness of prior training did not influence performance $(\mathrm{F}<1)$

Mean position judgments. Since the subjects used each number between 1 and 30 once on each temporal judgment test, mean judgments (as for the main effects of university and list) cannot be meaningfully compared. Only comparisons involving serial position are discussed here. As can be seen in Figure 2, judgments increased

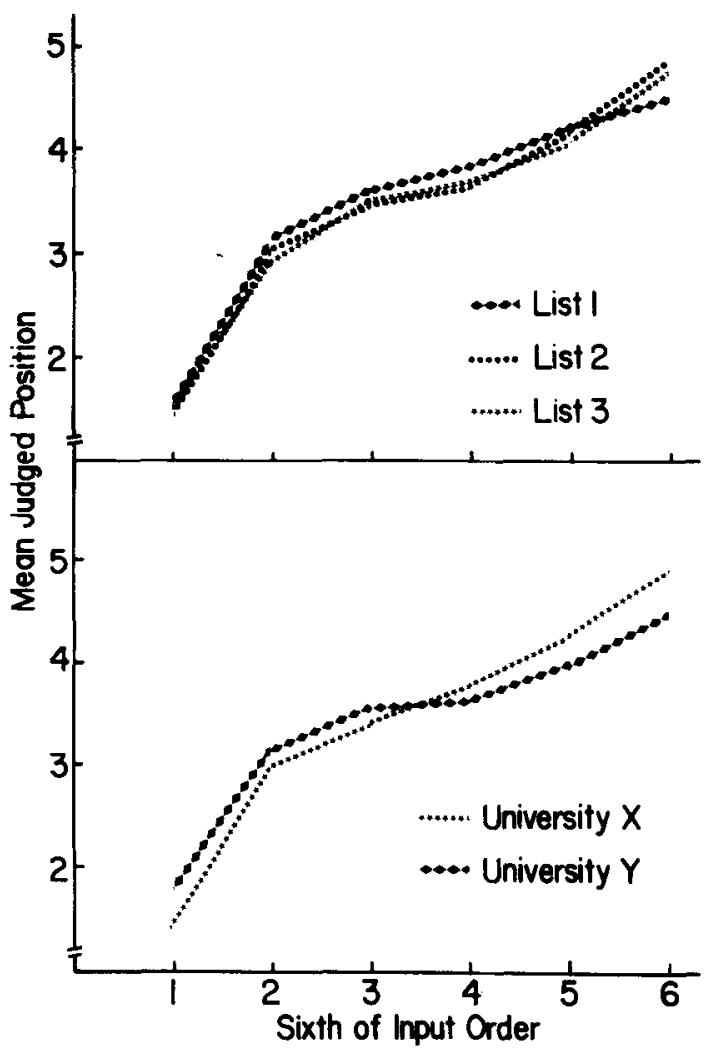

Figure 2. Mean position judgments on the temporal task as a function of list (top half) and of subject group (bottom half). (The numbers on the ordinate were computed by converting judgments of Positions 1-5 to 1s, of Positions 6-10 to 2s, etc.) reliably with input position $[\mathrm{F}(5,380)=357.21, \mathrm{MSe}=$ .70]. The slope of the function increased slightly from List 1 to List $3[\mathrm{~F}(10,760)=2.94, \mathrm{MSe}=.53]$. Finally, the slope was steeper for University $\mathrm{X}$ than for University $Y$ students $[F(5,380)=6.39, \mathrm{MSe}=.70]$.

For List 4 , the only significant effect was the serial position $x$ university interaction $[F(5,380)=4.79$, MSe $=.56]$; the form of this interaction was similar to that for Lists 1-3. Once more, there was no effect of appropriate versus inappropriate training on sensitivity to temporal order. In particular, the serial position $\mathrm{x}$ appropriateness interaction was not significant $[F(5,380)$ $=1.62, \mathrm{MSe}=.56]$.

Thus, the three measures of memory for temporal order gave fully consistent results: Accuracy of temporal knowledge improved with practice, and groups of students who differed in average academic ability showed differences in performance. These two findings are inconsistent with the automaticity hypothesis. The only finding consistent with the automaticity hypothesis is the lack of benefit for appropriate training seen on List 4: Practice at a recall task resulted in temporal performance that was equivalent to practice at a temporal judgment task. This may be because good performance at the temporal task benefits from encoding strategies similar to those used in free recall. The similar serial position curves on the two tasks (compare the top and bottom halves of Figure 1) and the strategy reports of the subjects in Groups T-T and T-R (to which we now turn) provide some support for common mnemonic strategies on the two tasks.

Strategy reports. Several different strategies were reported; these were difficult to categorize, in part because our questions failed to yield precise descriptions in many instances. However, it was clear from the reports that the subjects tended to abandon the strategy that they had tried on List 1 and to try out new strategies on later lists. On Lists 1 and 2, about $25 \%$ of the subjects reported attempting cumulative serial rehearsal of items as the items were presented. This strategy seems ideal for accurate encoding of temporal order, but it was too difficult for the subjects to use once several words had been shown, and thus was soon abandoned. On later lists, there was a trend toward use of strategies such as rehearsing words in pairs or in larger groups or making up sentences of successive sets of words. Although these latter strategies may not encode serial 
order as completely or as directly as serial rehearsal would seem to, they are more manageable and, critical for our interpretation of List 4 performance, they are not very different from what subjects are known to do on free-recall tasks (Rundus, 1971). Thus, practice at free recall is able to confer as much benefit on temporal task performance as practice at the temporal task itself. The reason that transfer from the temporal task to the free-recall task is not as complete as it is in the free recall to temporal direction is probably due to the fact that the temporal task provides no practice at retrieval, and efficient retrieval strategies, as well as good encoding strategies, are required for optimal free-recall performance.

\section{Interlist Correlations}

Each subject's performance on one list was correlated with his or her performance on the next list to determine whether the relative ordering of subjects was stable across lists. Since the Pearson product-moment correlations for University $\mathrm{X}$ and $\mathrm{Y}$ groups did not differ (all $\mathrm{Zs}<1.44$ ), only correlations for the combined groups are presented. Those for recall (shown in Table 4) were uniformly positive and significant. For the temporal task, interlist correlations were computed for both the correct judgments and the correlation between judged and true order measures. These correlations were somewhat lower than those for recall but still always positive and usually significant. They also tended to get larger over lists. $Z$ tests indicated that only the List 1 List 2 correlations were reliably higher for the recall test than for the temporal test.

Also computed were correlations between List 3 and List 4 performance for the two conditions in which the task changed for the last list. These correlations were not as high as the same-task correlations, but they were all significant $[\mathrm{r}(38)=.234-.286]$. And there were no differences between Conditions T-R and R-T. In general, then, the correlation data are consistent with the trends, suggested by the strategy reports and by the similar serial position effects, that with experience at a temporal

Table 4

Interlist Pearson Product-Moment Correlations for Recall and Temporal Performance

\begin{tabular}{llll}
\hline & \multicolumn{3}{c}{ Lists } \\
\cline { 2 - 4 } & \multicolumn{1}{c}{ Recall Task } \\
& $.663^{*}$ & $.601^{*}$ & $.614^{*}$ \\
Number Recalled & Temporal Task & & \\
& $.272^{* *}$ & $.517^{*}$ & $.657^{*}$ \\
Correct Judgments & .150 & $.428^{*}$ & $.382^{* *}$ \\
\hline
\end{tabular}

Note-For the first two columns, the correlations are for 80 sub. jects; for the last column, the correlations are based on the 40 subjects who continued with the same task.

${ }^{*} P<.01 . \quad * * p<.05$. task, subjects increase their use of encoding strategies typically associated with recall tasks.

Thus, the interlist correlations, like the rest of temporal task data, do not conform to the Hasher and Zacks (1979, in press) criteria of automaticity. The correlations indicate stable individual differences in performance on the temporal task; these were not expected on the basis of the automaticity hypothesis, but they are consistent with the differences in temporal performance between University $X$ and $Y$ students.

\section{DISCUSSION}

The free-recall results can be easily summarized. Recall benefited from appropriate practice. There were also stable individual differences in recall: (1) students who were good at recalling one list tended to be good at recalling others; and (2) students with higher academic skills also recalled more than students with lower skills. These results are consistent with the proposal that preparation for a free-recall test entails considerable effortful processing (see also Zacks et al., 1982).

The temporal results are more complex. It is clear that subjects are sensitive to temporal order information. Although this is in keeping with earlier research, as well as with the notion that temporal information might be processed automatically, the bulk of the findings contradict this viewpoint: (1) subjects got better with experience; (2) there were reliable individual and group differences in memory for temporal information; and (3) although the nature of prior training did not reliably influence performance on a final test, the trends were in the direction of superior performance by subjects with appropriate training. These findings are strikingly different from those obtained in our earlier research on encoding of frequency of occurrence. There, a design like the one used here yielded neither benefits from appropriate practice nor stable individual differences (Zacks et al., 1982, Experiment 1). Also, performance on a frequency task (but not on a free-recall task) was found to be invariant across groups of subjects who differed in academic achievement (Zacks et al., 1982, Experiment 3). It was concluded that these data supported the hypothesis that frequency information is encoded automatically. By contrast, the current results on encoding of temporal order yield a different conclusion. Because practice-related factors and individual differences (e.g., in motivation, in available capacity, and in knowledge of relevant strategies) seem to influence storage of temporal codes, we are led to the conclusion that the encoding of temporal information does not occur automatically, at least as that concept is defined by Hasher and Zacks (1979).

There is one methodological consideration that might call this conclusion into question: Specifically, the practice effects and individual differences might be due to performance factors associated with our measure 
of temporal encoding-perhaps practice and greater ability do not affect encoding at all, but instead benefit the retrieval of temporal order or the judging of serial position. Worries about the possible contaminating role of effortful performance factors at retrieval might seem particularly valid for a serial position judgment task such as ours. It requires subjects to think of multiple items at the same time, to assign a specific number to each item, and to keep track of the numbers already used. By contrast, a forced-choice recency-discrimination task seems much simpler in that it only requires subjects to think of two items at a time and to choose between them. The roles of such task variables have not been systematically evaluated, but previous research has suggested that it is not as important as might be expected. In general, different measures of temporal knowledge have yielded similar results with respect to automaticity criteria. For example, incidental encoding of temporal order information has been demonstrated both with a serial position judgment task (e.g., Hintzman \& Block, 1971) and with a forced-choice recencydiscrimination task (Tzeng \& Cotton, 1980). Also, two studies of aging effects on temporal coding, one of which used a serial position judgment task (McCormack, 1981) and the other of which used a forced-choice recency-discrimination task (Perlmutter et al., 1981), have agreed in the finding of no age deficit. Finally, one study (McCormack, 1982) that did obtain an age deficit in temporal coding used the apparently less effortful forced-choice procedure.

Such comparisons suggest that our findings are not tied to our specific test of memory for temporal order. Of course, these comparisons are cross-experimental ones, and therefore this argument does not rest on the firmest of empirical grounds. Still, we think it reasonable to take our results at face value as being contrary to the automaticity hypothesis.

The weight of the earlier evidence (e.g., Hintzman \& Block, 1971; McCormack, 1981; Perlmutter et al., 1981; Toglia \& Kimble, 1976) is in support of the automaticity hypothesis. Our results are at odds with this trend, and we now turn to the question of how all the data might be put together. One possible interpretation that would incorporate all the temporal results comes from the consideration that the Hasher and Zacks (1979) automaticity criteria were set for the anchor point of a continuum of encoding difficulty-the least capacity-demanding anchor point. By the rule of joint satisfaction of all the criteria, the frequency attribute can be said to lie at the minimum-capacity end (see Hasher \& Zacks, in press), but the temporal attribute cannot. Perhaps the temporal attribute falls somewhere away from this end and thus demands more capacity than frequency processing but less than, say, rehearsal. Such a view is consistent with the Hasher and Zacks framework (but not with the specific claims about temporal coding); it also is consistent with the body of data on temporal coding. However, it raises two problems. The first is the problem of measuring the capacity demands of mental operations that vary in their degree of nonautomaticity or effortfulness. Because Hasher and Zacks did not establish a ranking of importance for their various automaticity criteria, how to use these criteria to order operations when some, but not others, of the criteria are satisfied is not clear. Methodologies, such as probe reaction time, that attempt to measure directly the capacity required by different operations do not offer a simple solution; these also may have serious limitations (cf. Fisk et al., 1981). Thus, it may be difficult to test the hypothesis that encoding of temporal order is less than completely automatic.

The second problem with the less-than-completelyautomatic view is that it provides an incomplete account of temporal coding. For example, it leaves unanswered the question of which aspects of temporal coding are automatic and which are not. One model that may provide an at least partial solution to this problem is Tzeng's (Tzeng \& Cotton, 1980; Tzeng et al., 1979) "studyphase" retrieval model of temporal coding. Tzeng claimed that his model is an automaticity view, but, as will be seen, it incorporates some nonautomatic features. The model proposes that the process of study-phase retrieval is the basic mechanism by which encoding of the relative order of items occurs. For example, if, during the encoding of item $B$, the subject retrieves a previous item, item $A$, and rehearses items $A$ and $B$ together, then the mere fact that item $A$ is the remembered item and item B is the current one directly (i.e., automatically) establishes their correct relative order. The earlier data consistent with the Hasher and Zacks criteria (including those showing no intentionality effects) are explained by assuming (Tzeng \& Cotton, 1980) that the adult subjects of these experiments engage in displaced rehearsal whenever any retention test is forewarned or suspected. But, Tzeng's model can also accommodate data inconsistent with the Hasher and Zacks criteria (including those obtained here) by assuming differences in the amount of displaced rehearsal. Thus, our findings that there are benefits to temporal performance from practice and no differential transfer from appropriate versus inappropriate practice would follow from the assumption that displaced rehearsal increases over lists, and does so equally, whether the task involves free recall or temporal judgments. The latter suggestion is consistent with some of the strategies reported for the temporal task.

In sum, Tzeng's model seems to provide a useful first approximation to an account of both the automatic and the nonautomatic aspects of temporal coding. It does, however, need amplification to take care of some loose ends. For example, there is some evidence of equivalent temporal processing in groups that presumably differed in displaced rehearsals (Tzeng, 1976). Also, the model does not seem to account easily for good judgment of temporal order among recency items of a long list: Since these items are known to ordinarily 
have little rehearsal, they have little opportunity to enter into rehearsal sets, the necessary condition for temporal coding to occur. Although displaced rehearsal is probably an important mechanism by which temporal coding occurs (e.g., Johnson, 1983), it is undoubtedly not the only one. At the minimum, subjects also seem to use some mechanism that takes advantage of the anchor points of the beginning and the end of the list (e.g., Glanzer \& Dolinsky, 1965). In addition, there are a number of empirical loose ends in this area, not the least of which is the lack of systematic study of encoding versus retrieval effects on performance on various temporal tasks.

\section{REFERENCES}

Barron's Profiles of American Colleges (12th ed.) (1980). Woodbury, NY: Barron's Educational Series, Inc., College Division.

Battig, W. F., \& Montague, W. E. (1969). Category norms for verbal items in 56 categories: A replication and extension of the Connecticut category norms. Journal of Experimental Psychology Monographs, 80(3, Pt. 2).

Bellezza, F. S. (1982). Updating memory using mnemonic devices. Cognitive Psychology, 14, 301-327.

Brackbill, Y., \& Fitzgerald, H. E. (1972). Stereotype temporal conditioning in infants. Psychophysiology, 9, 569-577.

Bransford, J. D. (1979). Human cognition: Learning, understanding, and remembering. Belmont, CA: Wadsworth.

Brown, A. L. (1973). Judgments of recency for long sequences of pictures: The absence of a developmental trend. Journal of Experimental Child Psychology, 15, 473-480.

Fisk, A. D., Derrick, W. L., \& Schneider, W. (1981). The use of dual task paradigms in memory research: A methodological assessment and an evaluation of effort as a measure of levels of processing (Research Rep. No. HARL-ONR-8105). Champaign, IL: University of Illinois, Human Attention Research Laboratory.

Glanzer, M., \& Dolinsky, R. (1965). The anchor for the serial position effect. Journal of Verbal Learning and Verbal Behavior, 4, 267-273.

HAShe R, L. (1973). Position effects in free recall. American Journal of Psychology, 86, 389-397.

HAsher, L., \& ZACKs, R. T. (1979). Automatic and effortful processes in memory. Journal of Experimental Psychology: General, 108, 356-388.

Hasher, L., \& Zacks, R. T. (in press). Automatic processing of fundamental information: The case of frequency of occurrence. American Psychologist.

Hinrichs, J. V. (1970). A two-process memory-strength theory for judgment of recency. Psychological Review, 77, 223-233.

Hintzman, D. L., \& Block, R. A. (1971). Repetition and memory: Evidence for a multiple-trace hypothesis. Journal of Experimental Psychology, 88, 297-306.

Hintzman, D. L., \& BLock, R. A. (1973). Memory for the spacing of repetitions. Journal of Experimental Psychology, 99, 70-74.

Hirst, W. (1982). The amnesic syndrome: Descriptions and explanation. Psychological Bulletin, 91, 434-460.

Johnson, M. K. (1983). A multiple-entry, modular memory system. In G. H. Bower (Ed.), The psychology of learning and motivation: Advances in research and theory (Vol. 17, pp. 81-123). New York: Academic Press.
MCCormack, P. D. (1981). Temporal coding by young and elderly adults: A test of the Hasher-Zacks model. Developmental Psychology, 17, 509-515.

McCormack, P. D. (1982). Temporal coding and study-phase retrieval in young and elderly adults. Bulletin of the Psychonomic Society, 20, 242-244.

Navon, D., \& Gophen, D. (1979). On the economy of the humanprocessing system. Psychological Review, 86, 181-213.

Perlmutter, M., Metzger, R., Nezworski, T., \& Miller, K. (1981). Spatial and temporal memory in 20 and 60 year olds. Journal of Gerontology, 36, 59-65.

Proctor, R. W., \& Ambler, B. A. (1975). Effects of rehearsal strategy on memory for spacing and frequency. Journal of Experimental Psychology: Human Learning and Memory, 1, 640-647.

Rundus, D. (1971). Analysis of rehearsal processes in free recall. Journal of Experimental Psychology, 89, 63-77.

Toglia, M. P., \& Kimble, G. A. (1976). Recall and use of serial position information. Journal of Experimental Psychology: Human Learning and Memory, 2, 431-445.

Tulving, E., \& Madigan, S. A. (1970). Memory and verbal learning. Annual Review of Psychology, 21, 437-484.

Tzeng, O. J. L. (1976). A precedence effect in the processing of verbal information. American Journal of Psychology, 89, 577-599.

Tzeng, O. J. L., \& Cotron, B. (1980). A study-phase retrieval model of temporal coding. Journal of Experimental Psychology: Human Learning and Memory, 6, 705-716.

Tzeng, O. J. L., Lee, A. T., \& Wetzel, C. D. (1979). Temporal coding in verbal information processing. Journal of Experimental Psychology: Human Learning and Memory, 5, 52-64.

Unde Rwood, B. J. (1949). Proactive inhibition as a function of time and degree of prior learning. Journal of Experimental Psychology, 39, 24-34.

UNDE RWOOD, B. J., \& MALMI, R. A. (1978). An evaluation of measures used in studying temporal codes for words within a list. Journal of Verbal Learning and Verbal Behavior, 17, 279-293.

WinOGRAD, E. (1968). List differentiation as a function of frequency and retention interval. Journal of Experimental Psychology Monographs, 76(2, Pt. 2).

Zacks, R. T., Hasher, L., \& SanfT, H. (1982), Automatic encoding of event frequency: Further findings. Journal of Experimental Psychology: Learning, Memory, and Cognition, 8, $106-116$.

Zimmerman, J., \& Underwood, B. J. (1968). Ordinal position knowledge within and across lists as a function of instructions in free-recall learning. Journal of General Psychology, 79, 301-307.

\section{NOTES}

1. This information comes from Barron's Profiles of American Colleges (12th ed.) (1980).

2. Another measure that has sometimes been used with temporal judgment tasks is the Adjusted Index of Location (AIL), developed by Toglia and Kimble (1976). A presumed advantage of the AIL measure is that it corrects for the known bias of guessing midlist locations when the correct location cannot be recalled. Since the procedures of the current experiment prevented this bias from operating (each location could be used only once on each test), AIL scores were not computed for our data.

(Manuscript received July 26, 1983 ; revision accepted for publication March 29,1984 .) 